\title{
Utilization of sputter depth profiling for the determination of band alignment at polycrystalline CdTe/CdS heterointerfaces
}

Cite as: Appl. Phys. Lett. 81, 2297 (2002); https://doi.org/10.1063/1.1507830

Submitted: 18 February 2002 • Accepted: 23 July 2002 • Published Online: 09 September 2002

J. Fritsche, T. Schulmeyer, D. Kraft, et al.

ARTICLES YOU MAY BE INTERESTED IN

First-principles calculation of band offsets, optical bowings, and defects in CdS, CdSe, CdTe, and their alloys

Journal of Applied Physics 87, 1304 (2000); https://doi.org/10.1063/1.372014

Thin-film CdS/CdTe solar cell with $15.8 \%$ efficiency

Applied Physics Letters 62, 2851 (1993); https://doi.org/10.1063/1.109629

Emitter/absorber interface of CdTe solar cells

Journal of Applied Physics 119, 233104 (2016); https://doi.org/10.1063/1.4953820

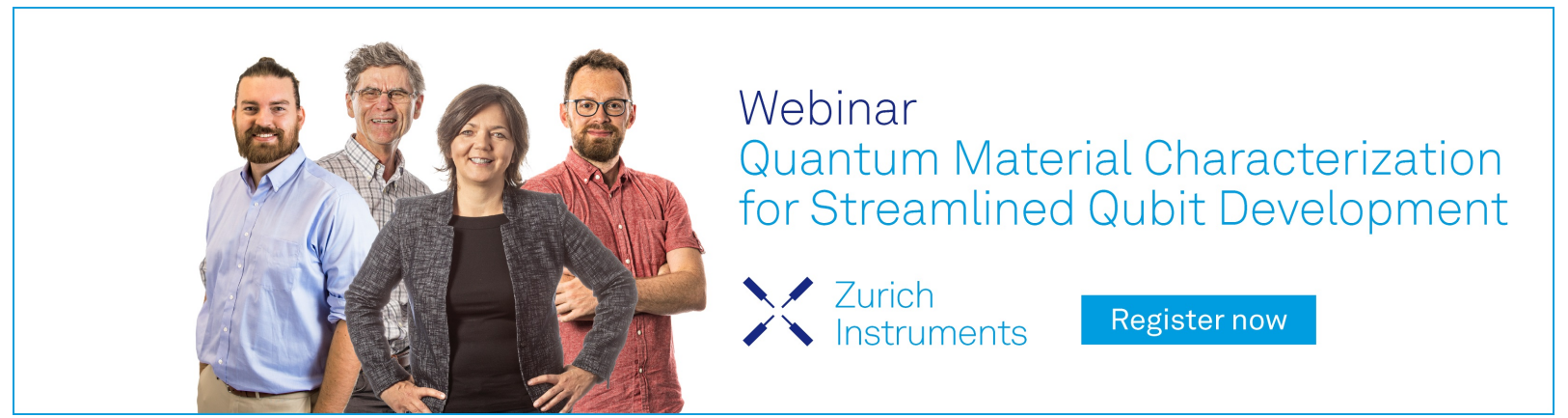




\title{
Utilization of sputter depth profiling for the determination of band alignment at polycrystalline $\mathrm{CdTe} / \mathrm{CdS}$ heterointerfaces
}

\author{
J. Fritsche, T. Schulmeyer, D. Kraft, A. Thißen, A. Klein, ${ }^{a)}$ and W. Jaegermann \\ Darmstadt University of Technology, Institute of Materials Science, Surface Science Division, \\ Petersenstrasse 23, D-64287 Darmstadt, Germany
}

(Received 18 February 2002; accepted for publication 23 July 2002)

\begin{abstract}
The band alignment at polycrystalline $\mathrm{CdS} / \mathrm{CdTe}$ heterointerfaces for thin-film solar cells is determined by photoelectron spectroscopy from stepwise CdTe deposition on polycrystalline CdS substrates and from subsequent sputter depth profiling. Identical values of $0.94 \pm 0.05 \mathrm{eV}$ for the valence band offset are obtained. (c) 2002 American Institute of Physics.
\end{abstract}

[DOI: $10.1063 / 1.1507830]$

It is generally believed that the rectifying contact in the CdTe thin film solar cell is formed by a $n-\mathrm{CdS} / p-\mathrm{CdTe}$ junction. ${ }^{1}$ A large built-in voltage in the CdTe layer and a low barrier for the electrons at this interface are ideal for maximum conversion efficiency which requires a small conduction band discontinuity. The band alignment at semiconductor heterojunctions can be determined using electrical techniques, internal photoemission and photoelectron spectroscopy (PES). ${ }^{2}$ Photoelectron spectroscopy is widely used for this application as it allows for parallel determination of chemical and electronic interface properties, which is the basis for studying fundamental properties and the possibilities of modifying semiconductor heterojunctions. ${ }^{3}$

A typical PES experiment for the determination of band alignments is performed by stepwise deposition of the film material on a clean substrate surface. Both substrate and film preparation are done in ultrahigh vacuum (UHV) systems, which are directly connected to the photoelectron spectrometer to avoid sample contaminations. The preparation of semiconductor interfaces by stepwise deposition in UHV is often far from the actual manufacturing technology of empirically optimized devices. In the case of the CdTe thinfilm solar cell the CdTe and CdS films are usually prepared either by electrochemical deposition or by a very fast deposition at high substrate temperatures using close-spaced sublimation. ${ }^{1,4}$ Sufficient efficiencies are only achieved after a chlorine treatment, the so-called activation. ${ }^{1,5}$ To understand the effect of activation on the electronic properties of the $\mathrm{CdTe} / \mathrm{CdS}$ heterojunction, an investigation of an activated interface is required. This is usually not feasible with surface sensitive techniques such as photoemission as the interface is buried below a thick CdTe layer. One possibility for the determination of band alignment at buried interfaces is sputter depth profiling. However, it is well known that sputtering is highly species sensitive leading to preferential sputtering of compound materials. This will generally modify the electronic structure of the materials and hence hinders the determination of electronic properties. Sputter depth profiling is therefore not an accepted procedure for the determination of band alignment at buried interfaces.

In this letter the determination of the band alignment at

a)Electronic mail: aklein@surface.tu-darmstadt.de the $\mathrm{CdTe} / \mathrm{CdS}$ heterointerface by photoelectron spectroscopy is described. The interface properties are investigated by deposition of CdTe on an evaporated CdS film step by step on one sample without breaking the vacuum as well as by subsequent sputter depth profiling of the same sample. The experiments were performed using a Physical Electronics PHI 5700 electron spectrometer system equipped with a monochromated Al $K \alpha$ x-ray source, a He discharge lamp, and a focusing sputter gun. X-ray photoelectron spectra were taken with $45^{\circ}$ off-normal emission. He I excited valence band spectra [ultraviolet photoelectron spectroscopy (UPS)] were taken in normal emission with an applied sample bias voltage of $-1.5 \mathrm{~V}$. Sputtering was performed with $1 \mathrm{keV}$ argon ions at $0.05 \mathrm{~mA} / \mathrm{cm}^{2}\left(\sim 3 \times 10^{14}\right.$ ions $\left./ \mathrm{cm}^{2} \mathrm{~s}\right)$ giving a typical sputter rate of $\sim 6 \AA / \mathrm{min}$. The deposition chamber for CdS and CdTe was directly attached to the surface analysis system. Films are deposited by thermal evaporation of materials with $99.99 \%$ purity from $\mathrm{Al}_{2} \mathrm{O}_{3}$ crucibles using deposition rates of $3 \AA$ Amin at source temperatures of $500^{\circ} \mathrm{C}$ for $\mathrm{CdTe}$ and $600^{\circ} \mathrm{C}$ for $\mathrm{CdS}$. Film thicknesses were determined using a quartz microbalance. As substrates indium tin oxide/ $\mathrm{SnO}_{2}$ coated glass as used for solar cell manufacturing were used. Prior to deposition of thick CdS films the substrates were cleaned by extensive rinsing in alcohol and deionized water and subsequent heating in UHV. All deposition steps were performed at room temperature.

In Fig. 1 we show S $2 p$ and $\mathrm{Te} 4 d$ core level lines obtained after stepwise deposition of CdTe on CdS up to 16 min corresponding to a CdTe film thickness of $48 \AA$. The $\mathrm{S}$ $2 p$ emission lines are gradually decreased with increasing film thickness. The attenuation with film thickness corresponds to a layer-by-layer growth mode. No chemical components other than those attributed to $\mathrm{CdTe}$ and $\mathrm{CdS}$ can be identified. The difference in $\mathrm{S} 2 p$ and Te $4 d$ core level binding energy is given by $E_{B}\left(\mathrm{~S} 2 p_{3 / 2}\right)-E_{B}\left(\mathrm{Te} 4 d_{5 / 2}\right)=121.57$ $\pm 0.02 \mathrm{eV}$ (see also Fig. 3).

$\mathrm{S} 2 p$ and Te $4 d$ core level lines obtained during sputter depth profiling of the CdTe/CdS interface are shown in Fig. 2. The same sample as shown in Fig. 1 has been used. With increasing sputtering time the Te $4 d$ intensity decreases and the S $2 p$ intensity increases. There are no indications for chemically shifted components and the linewidths of the spectra are comparable to those shown in Fig. 1. 


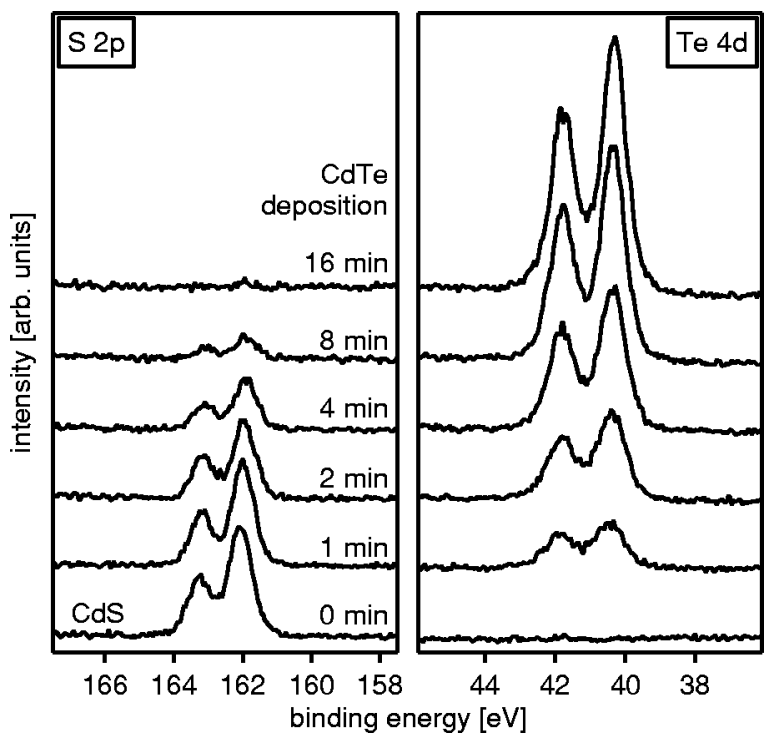

FIG. 1. S $2 p$ and Te $4 d$ core level lines obtained during stepwise deposition of CdTe on CdS. The deposition rate was $3 \AA / m i n$.

The binding energy difference between $\mathrm{S} 2 p$ and Te $4 d$ determined for the deposition and sputter depth profile is given in Fig. 3. At the beginning and the end of the sputter series there is an increased scatter in the binding energy differences which is due to nonaccurate binding energy determination as a result of the low photoemission intensities of the $\mathrm{S} 2 p$ and the Te $4 d$ line, respectively. For intermediate sputtering times there is enough intensity for both core levels for accurate binding energy determination. The binding energy difference is then given by $E_{B}\left(\mathrm{~S} 2 p_{3 / 2}\right)-E_{B}\left(\mathrm{Te} 4 d_{5 / 2}\right)$ $=121.55 \pm 0.03 \mathrm{eV}$, which agrees with the value determined during the stepwise growth of the interface.

To determine the valence band offset the binding energies of the core levels with respect to the valence band maximum of bulk materials have to be known in addition. The values have been determined from a number of films as $E_{B}^{\mathrm{VBM}}=159.97 \pm 0.05 \mathrm{eV}$ for $\mathrm{S} 2 p$ in $\mathrm{CdS}$ and $E_{B}^{\mathrm{VBM}}$ $=39.35 \pm 0.05 \mathrm{eV}$ for Te $4 d$ in CdTe. ${ }^{6}$ With an average core

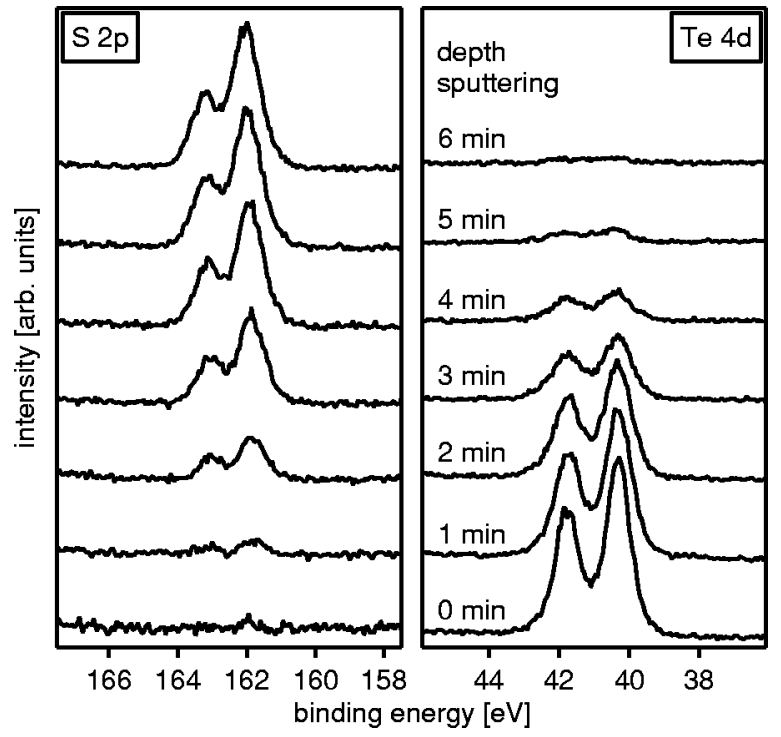

FIG. 2. S $2 p$ and Te $4 d$ core level lines obtained during sputter depth profiling of CdTe on CdS.

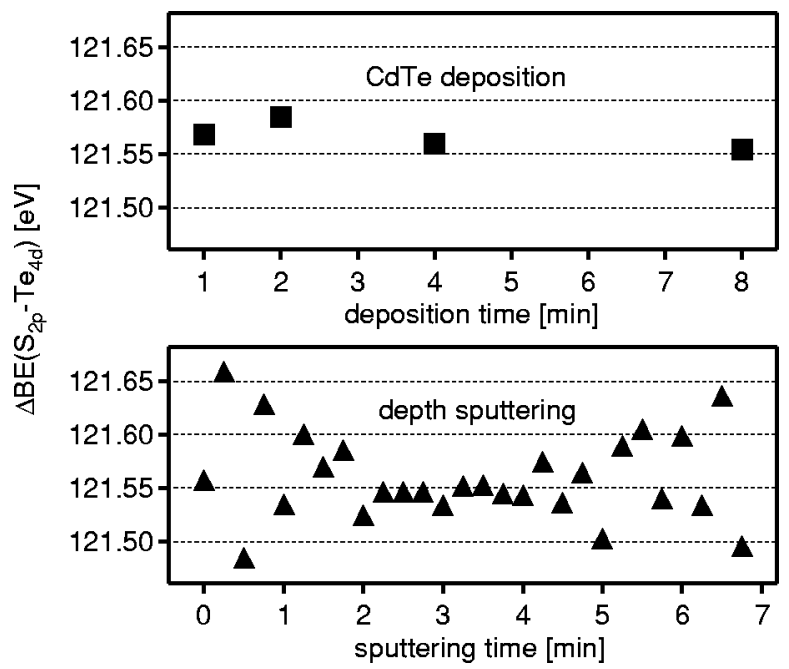

FIG. 3. Binding energy differences between the S $2 p$ and Te $4 d$ core-level lines for stepwise deposition of CdTe on CdS and subsequent sputter depth profiling.

level binding energy difference of $121.56 \mathrm{eV}$, the valence band discontinuity is then determined as $\Delta E_{\mathrm{VB}}=0.94$ $\pm 0.05 \mathrm{eV}$. Together with the band gaps of CdTe and CdS of 1.45 and $2.42 \mathrm{eV}$, respectively, this corresponds to a conduction discontinuity of $\Delta E_{\mathrm{CB}}=0.03 \pm 0.05 \mathrm{eV}$. The resulting energy band diagram is presented in Fig. 4. The position of the vacuum level is determined from work function measurements using UPS.

Considering that the conduction bands in tetrahedral semiconductors have mainly cationic character, an estimation based on the common anion (cation) rule ${ }^{7}$ would suggest a small conduction band offset because $\mathrm{Cd}$ is present in both semiconductors. However, present theories of semiconductor band alignment indicate a general failure of the common anion rule to predict a correct band alignment (see, e.g., Refs. 3, 8, and 9 and references therein). Using Harrison's tight binding theory including interface dipoles, ${ }^{10}$ the valence band offset is determined by alignment of the average hybrid orbital energies to be $\Delta E_{\mathrm{VB}}=0.71 \mathrm{eV}$. Spin-orbit coupling, which shifts the valence band maximum upwards by $\Delta_{s o} / 3$, is significant only for CdTe. ${ }^{11}$ The valence band offset including spin-orbit coupling is then given by $\Delta E_{\mathrm{VB}}=0.71$ $+0.28=0.99 \mathrm{eV}$. An identical value of $\Delta E_{\mathrm{VB}}=0.99 \mathrm{eV}$ is determined by Wei et al. using a first principles supercell calculation of the natural (unstrained) band alignment for the $\mathrm{CdS} / \mathrm{CdTe}$ interface. ${ }^{12}$ Tersoff's branch point energy mode ${ }^{13}$ or the dielectric midgap energy model of Cardona and

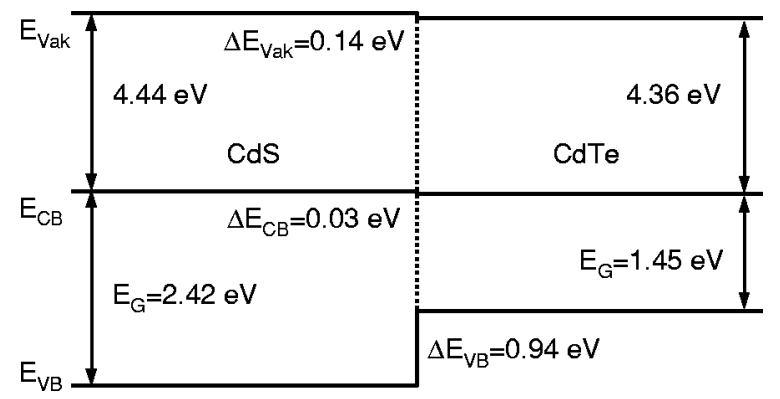

FIG. 4. Schematic energy band diagram of the polycrystalline CdS/CdTe heterojunction as determined from data in Figs. 1 and 2. 
Christensen ${ }^{14}$ cannot be used here for comparison, because they give only values for zinc blende compounds and not for CdS, whose stable crystallographic structure is wurtzite.

All theoretical models, however, have to be modified when strain is present in either or both of the semiconductors, because the electronic structure around the band edges will be modified. ${ }^{15}$ The lattice mismatch at the CdS/CdTe interface amounts to $\epsilon=\left(a_{\mathrm{CdTe}}-a_{\mathrm{CdS}}\right) / a_{\mathrm{CdS}}=11.4 \%$. For such large lattice mismatch the critical film thickness, up to which defect free strained layers can exist, should be of the order of the lattice constant. It is thus most likely that the mismatch is relieved by the formation of interfacial defects as observed for a number of such highly mismatched materials combinations (see, e.g., Refs. 16 and 17). The agreement of the experimental valence band offset determined here with the available theoretical estimates and calculations for (unstrained) interfaces supports this assumption.

An experimental determination of the band offset at the $\mathrm{CdS} / \mathrm{CdTe}$ interface using photoelectron spectroscopy has been presented by Niles and Höchst. ${ }^{18} \mathrm{CdS}$ was evaporated on (UHV) cleaved $\mathrm{CdTe}(110)$ surfaces at a substrate temperature of $T=250^{\circ} \mathrm{C}$ resulting in pseudomorphic growth of cubic CdS and a valence band offset of $\Delta E_{\mathrm{VB}}=0.65 \mathrm{eV}$, which differs from the present result. A theoretical calculation including strain yielded $\Delta E_{\mathrm{VB}}=1.19 \mathrm{eV} .{ }^{18}$ Hence strain hardly accounts for the difference between the present result and the experimental value given by Niles and Höchst. There is also only a weak dependence of the band alignment on interface orientation with values for $\Delta E_{\mathrm{VB}}$ close to $1 \mathrm{eV}$ as determined previously, ${ }^{6}$ which agrees with expectations for unstrained interfaces between homovalent semiconductors. ${ }^{19,20}$ A further determination of the conduction band offset of polycrystalline $\mathrm{CdS} / \mathrm{CdTe}$ interfaces was given by Nishi et al. using internal photoemission induced by tunable infrared radiation from a free electron laser. ${ }^{21}$ Their result $\left(\Delta E_{\mathrm{CB}} \sim 100 \mathrm{meV}\right)$ is also close to the band alignment presented here. The deviation of the value given by Niles and Höchst is still unclear and requires more systematic investigations.

In summary, we have determined the band alignment at polycrystalline $\mathrm{CdTe} / \mathrm{CdS}$ heterojunctions using in situ pho- toelectron spectroscopy of stepwise deposited and sputter depth profiled interfaces. The determined band alignment is the same in both experiments and agrees with theoretical predictions and also with other experiments. This indicates that sputter depth profiling does not lead to a modification of this particular interface. Preferential sputtering probably does not play any role because of either the mild sputtering conditions and the similar atomic masses of $\mathrm{Cd}$ and $\mathrm{Te}$. The possibility of achieving reasonable experimental band offsets by sputter depth profiling in the CdTe/CdS system, in combination with UHV processing of model structures, allows us to investigate the effects of solar cell processing, like, e.g., the $\mathrm{CdCl}_{2}$ treatment, on the electronic properties of buried heterointerfaces.

${ }^{1}$ R. H. Bube, Photovoltaic Materials (Imperial College Press, London, 1998).

${ }^{2}$ F. Capasso and G. Margaritondo, Heterojunction Band Discontinuities (North-Holland, Amsterdam, 1987).

${ }^{3}$ A. Franciosi and C. G. Van de Walle, Surf. Sci. Rep. 25, 1 (1996).

${ }^{4}$ K. Zweibel and R. Mitchell, Adv. Sol. Energy 6, 485 (1990).

${ }^{5}$ B. E. McCandless, I. Youm, and R. W. Birkmire, Prog. Photovoltaics 7, 21 (1999).

${ }^{6}$ J. Fritsche, A. Thissen, A. Klein, and W. Jaegermann, Thin Solid Films 387, 158 (2001).

${ }^{7}$ J. O. McCaldin, T. C. McGill, and C. A. Mead, Phys. Rev. Lett. 36, 56 (1976).

${ }^{8}$ J. Tersoff, Phys. Rev. Lett. 56, 2755 (1986.)

${ }^{9}$ E. T. Yu, J. O. McCaldin, and T. C. McGill, Band Offsets in Semiconductor Heterojunctions, in Solid State Physics, edited by H. Ehrenreich and D. Turnbull (Academic, Boston, 1992), Vol. 46, pp. 1-146.

${ }^{10}$ W. A. Harrison and J. Tersoff, J. Vac. Sci. Technol. B 4, 1068 (1986).

${ }^{11}$ O. Madelung, Semiconductors Basic Data (Springer, Berlin, 1996).

${ }^{12}$ S. -H. Wei, S. B. Zhang, and A. Zunger, J. Appl. Phys. 87, 1304 (2000).

${ }^{13}$ J. Tersoff, Phys. Rev. B 30, 4874 (1984).

${ }^{14}$ M. Cardona and N. E. Christensen, Phys. Rev. B 35, 6182 (1987).

${ }^{15}$ E. P. O'Reilly, Semicond. Sci. Technol. 4, 121 (1989).

${ }^{16}$ G. Bratina, L. Sorba, A. Antonini, R. Nicolini, G. Biasiol, A. Franciosi, J. E. Angelo, and W. W. Gerberich, Phys. Rev. B 48, 8899 (1993).

${ }^{17}$ M. Horn-von Hoegen, A. Al-Falou, B. H. Müller, and M. Henzler, Surf. Sci. 298, 29 (1993).

${ }^{18}$ D. W. Niles and H. Höchst, Phys. Rev. B 41, 12710 (1990).

${ }^{19}$ W. R. L. Lambrecht and B. Segall, Phys. Rev. B 41, 2832 (1990).

${ }^{20}$ R. G. Dandrea, C. B. Duke, and A. Zunger, J. Vac. Sci. Technol. B 10, 1744, (1992).

${ }^{21}$ K. Nishi, H. Ohyama, T. Siuzuki, T. Mitsuyu, and T. Tomimasu, Appl. Phys. Lett. 70, 3585 (1997).

This article may be downloaded for personal use only. Any other use requires prior permission of the author and AIP Publishing. This article appeared in Appl. Phys. Lett. 81, 2297 (2002) and may be found at https://doi.org/10.1063/1.1507830.

Available under only the rights of use according to UrhG. 